\title{
Neutron Crystal-Field Spectroscopy in Underdoped and Overdoped Copper Oxide Superconductors
}

\author{
Abert Furrer,' Joël Mesot,' Wolfgang Henggeler,' and Grit Böttger'
}

Received 9 December 1996

\begin{abstract}
In many cuprate superconductors rare-earth ( $R$ ) ions can be placed close to the superconducting copper oxide planes; thus, the crystal-field interaction at the $\mathrm{R}$ site constitutes an ideal probe of the local symmetry as well as the local charge distribution and thereby monitors directly changes of the carrier concentration induced by doping. For several compounds the crystal-field spectra observed by inelastic neutron scattering separate into different local components whose spectral weights distinctly depend on the doping level, i.e., there is clear experimental evidence for the formation of clusters which make the systems inhomogeneous. Moreover, it is found that the intrinsic linewidths of crystal-field transitions vary with temperature, which is essentially a reflection of the density of states associated with the charge carriers at the Fermi energy. Linewidth studies can therefore reveal information about the energy gap. For underdoped systems there is evidence for the formation of a pseudogap at $T^{*}>T_{c}$.
\end{abstract}

KEY WORDS: Neutron spectroscopy; cuprates; crystal field; inhomogeneous materials properties; energy gap; pseudogap.

\section{INTRODUCTION}

High-temperature superconductors are basically different from classical superconducting compounds in the sense that superconductivity is usually realized through doping, i.e., the homogeneous parent compound is structurally distorted by the doping elements. As a consequence high-temperature superconductors are intrinsically inhomogeneous, and the superconductivity is realized through a percolation process [1]. Such inhomogeneities have actually been observed by a variety of experiments [2] and most convincingly by local techniques such as Mössbauer spectroscopy [3], muon spin rotation [4], NMR spectroscopy [5], and EXAFS experiments [6]. In this paper we discuss an alternative local technique which is based on the fact that in most cuprate superconductors rare-earth $(\mathrm{R})$ ions can be introduced into the system close to the superconducting $\mathrm{CuO}_{2}$ planes; thus, the crystal-field interaction at the $\mathrm{R}$ site constitutes an ideal probe of the local symmetry as well as

'Laboratory for Neutron Scattering, ETH Zurich and Paul Scherrer Institute, CH-5232 Villigen PSI, Switzerland. the local charge distribution and thereby monitors directly changes of the carrier concentration induced by doping. Neutron spectroscopy is particularly suited to observe crystal-field effects in the optically opaque cuprate systems. Moreover, as the relaxation behavior of crystal-field exitations is predominantly governed by the interaction with the charge carriers, their intrinsic linewidths reflect the density of states at the Fermi energy similar to nuclear magnetic resonance experiments which reveal information on the energy gap. In neutron scattering experiments, however, the local susceptibility $\chi(q, \omega)$ is probed on a meV energy scale, i.e., many orders of magnitude larger than in NQR and NMR experiments.

In Section 2 we give an example of the cluster formation in underdoped $\mathrm{Pr}_{2-x} \mathrm{Ce}_{x} \mathrm{CuO}_{4-\delta}$. Section 3 presents neutron spectroscopic results obtained for $\mathrm{Er}_{x} \mathrm{Ca}_{1-x} \mathrm{Ba}_{2} \mathrm{Cu}_{3} \mathrm{O}_{7-o}$ which provides the first experimental evidence for cluster formation in an overdoped compound. In Section 4 we discuss the relaxation behavior of crystal-field excitations in the slightly underdoped compounds $\mathrm{HoBa}_{2} \mathrm{Cu}_{4} \mathrm{O}_{8}$ and $\mathrm{Er}_{2} \mathrm{Ba}_{4}$ $\mathrm{Cu}_{7} \mathrm{O}_{15}$ and compare our results with similar data 
obtained for optimally doped $\mathrm{Ho}_{0.1} \mathrm{Y}_{0.9} \mathrm{Ba}_{2} \mathrm{Cu}_{3} \mathrm{O}_{7-\delta}$ [7]. We find that for underdoped compounds a pseudogap is formed in the normal state at $T^{*}>T_{c}$ which was also reported from ARPES experiments performed for $\mathrm{Bi}_{2} \mathrm{Sr}_{2} \mathrm{CaCu}_{2} \mathrm{O}_{8+\delta}$ [8]. The currently controversial situation with the superconducting energy gap, the pseudogap, and the spin gap is discussed in Section 5.

\section{UNDERDOPED $\operatorname{Pr}_{2-x} \mathrm{Ce}_{x} \mathrm{CuO}_{4-\infty}$}

In $\mathrm{Pr}_{2-x} \mathrm{Ce}_{x} \mathrm{CuO}_{4-\delta}$ the crystal-field interaction of tetragonal symmetry splits the ground-state $J$ multiplet ${ }^{3} \mathrm{H}_{4}$ of the $\mathrm{Pr}^{3+}$ ions into a singlet ground state $\Gamma_{4}$, a first excited doublet $\Gamma_{5}$ at $18 \mathrm{meV}$, and further excited states at around $80 \mathrm{meV}$ [9]. Upon doping with $\mathrm{Ce}^{4+}$ ions the crystal-field transitions exhibit substructures which are most pronounced for the lowest excitation $\Gamma_{4} \rightarrow \Gamma_{5}[10]$. As shown in Fig. 1 for $x=0.10$ the $\Gamma_{4} \rightarrow \Gamma_{5}$ transition can be decomposed into four lines. The crystal-field transitions $A, B$ ( $=B_{1}+B_{2}$, splitting due to local symmetry which is lower than tetragonal), and $\mathrm{C}$ can be associated with undoped, intermediately doped, and highly doped character, respectively. For a critical volume fraction of 50\% the doped regions are infinitely connected by a two-dimensional percolative network [11], and the system is predicted to undergo a transition from the insulating to the metallic state (with $T_{c} \approx 25 \mathrm{~K}$ ) for $x \approx 0.14$ [10], in excellent agreement with the observed onset of superconductivity. Our neutron spectroscopic results have been confirmed by Raman experiments [12].

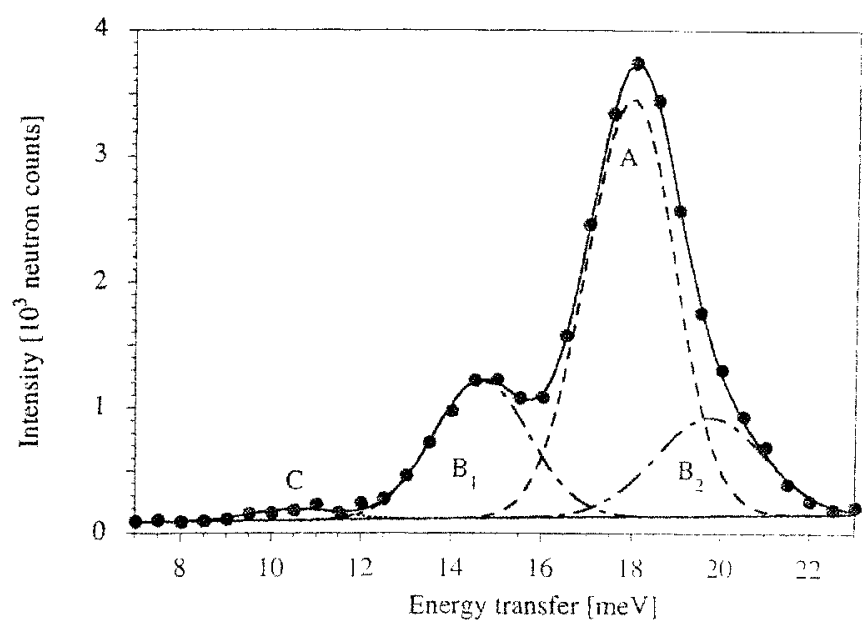

Fig. 1. Low-energy part of the neutron spectrum observed for $\operatorname{Pr}_{1.90} \mathrm{Ce}_{0.10} \mathrm{CuO}_{4-\delta}$ at $T=10 \mathrm{~K}[10]$. The lines denote the decomposition into individual Gaussian peaks.
It is important to note that diffraction techniques indicate a tetragonal symmetry for $\mathrm{Pr}_{2-x} \mathrm{Ce}_{x} \mathrm{CuO}_{4-s}$ independent of the doping level. However, local probes demonstrate that the symmetry of the doped clusters is lower than tetragonal as clearly evidenced by the splitting of the $\Gamma_{4} \rightarrow \Gamma_{5}$ transition $A$ (undoped regions with tetragonal symmetry) into two lines $B_{1}$ and $B_{2}$ (intermediately doped regions with lower symmetry).

\section{OVERDOPED $\mathrm{Er}_{1-x} \mathrm{Ca}_{x} \mathrm{Ba}_{2} \mathrm{Cu}_{3} \mathrm{O}_{7-s}$}

In $\mathrm{ErBa}_{2} \mathrm{Cu}_{3} \mathrm{O}_{7-\delta}$ the crystal-field interaction splits the ground-state $J$-multiplet ${ }^{4} I_{15 / 2}$ of the $\mathrm{Er}^{3+}$ ions into eight Kramers doublets. The detailed crystal-field level structure has been established by neutron spectroscopy [13]. The three low-lying crystalfield transitions observed for $\delta=0.08\left(T_{c}=92 \mathrm{~K}\right)$ are shown in Fig. 2a. Upon oxygen reduction the energies of the lines $B$ and $C$ remain roughly unaffected, whereas the line A shifts markedly to lower energy [13]. Moreover, the transition A splits into three different lines [14] which originate from different local environments of the $\mathrm{Er}^{3+}$ ions, i.e., there is clear evidence for the formation of clusters (similar to

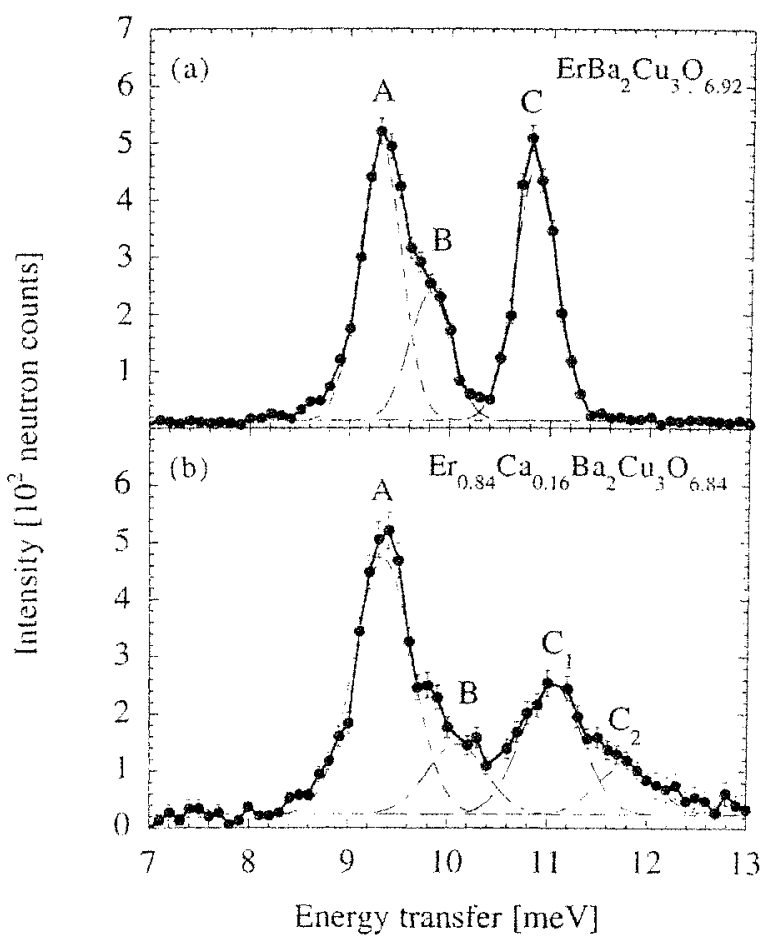

Fig. 2. Energy spectra of neutrons scattered from (a) $\mathrm{ErBa}_{2} \mathrm{Cu}_{3} \mathrm{O}_{6.98}$ [14] and (b) $\mathrm{Er}_{0.84} \mathrm{Ca}_{40.16} \mathrm{Ba}_{2} \mathrm{Cu}_{3} \mathrm{O}_{6.84}$ at $T=10 \mathrm{~K}$. The lines are as in Fig. 1. 
$\operatorname{Pr}_{2-x} \mathrm{Ce}_{x} \mathrm{CuO}_{4-\delta}$, see Section 2). The volume fractions of the three cluster types vary distinctly with oxygen reduction and correctly predict the critical oxygen contents associated with the two-plateau structure of $T_{c}$ in terms of two-dimensional bond percolation [13].

Optimum doping for the $\mathrm{RBa}_{2} \mathrm{Cu}_{3} \mathrm{O}_{7-\delta}$ compounds is realized for $\delta \approx 0.05$ [15]. The stoichiometric compounds are therefore slightly overdoped. Partial substitution for $\mathrm{R}^{3+}$ with $\mathrm{Ca}^{2+}$ makes these systems accessible far into the overdoped region [16]. This motivated us to study the crystal-field interaction in the compound series $\mathrm{Er}_{1-x} \mathrm{Ca}_{x} \mathrm{Ba}_{2} \mathrm{Cu}_{3} \mathrm{O}_{7-\delta}$ $(0 \leq x \leq 0.2$ with $\Delta x=0.04 ; \delta \leq 0.16)$. The inelastic neutron scattering experiments were performed with use of the triple-axis spectrometer IN3 at the high-flux reactor of the Institute Laue-Langevin at Grenoble. A typical energy spectrum covering the ground-state transitions to the three lowest crystal-field states of $\mathrm{Er}_{0.84} \mathrm{Ca}_{016} \mathrm{Ba}_{2} \mathrm{Cu}_{3} \mathrm{O}_{7-\delta}\left(\delta=0.16, T_{c}=62 \mathrm{~K}\right)$ is shown in Fig. 2b. While the energies and intensities of the crystal-field transitions $A$ and $B$ are independent of the $\mathrm{Ca}$ doping (see Fig. 2a), the crystal-field excitation $\mathrm{C}$ is clearly split into two components. More specifically, upon raising the $\mathrm{Ca}$ concentration the intensities of the lines $C_{1}$ and $C_{2}$ are decreasing and increasing, respectively. Obviousiy, the strengths of the crystalfield transitions $C_{1}$ and $C_{2}$ are directly related to the volume fractions of the optimally doped and overdoped regions, respectively. We have analyzed the observed energy spectra separately for the optimally doped and overdoped clusters on the basis of a crystal-field Hamiltonian of orthorhombic symmetry [13] and found that the crystal-field potential of $\mathrm{Er}^{3+}$ is reduced by $2 \%$ for the overdoped regions as compared to the optimally doped regions. This difference can be explained by the slight increase of the interatomic Er$O(2,3)$ distances [16] as well as the increased number of carriers associated with the overdoped regions.

\section{LINEWIDTH OF CRYSTAL-FIELD TRANSITIONS}

The linewidth of crystal-field excitations in metallic systems is mainly governed by relaxation effects with the charge carriers. For superconducting materials the temperature dependence of the linewidth therefore yields information on the energy gap $\Delta$, provided the excitation energies $h \omega<2 \Delta$. Crystal-field relaxation studies are similar to the most prominent relaxation methods NMR and NQR; however, crystal-field relaxation has the advantage to work in zero magnetic field (NMR needs a static external field) and at $\mathrm{THz}$ frequencies (NMR and NQR work at $\mathrm{MHz}$ frequencies). The linewidth of a transition with energy $h \omega_{i j}$ between the crystal-field states $|i\rangle$ and $|j\rangle$ is [17]

$$
\begin{aligned}
\Gamma_{i j}= & 2 J_{\mathrm{ex}}^{2}\left[M_{i j}^{2} \operatorname{coth}\left(h \omega_{i j} / 2 k_{B} T\right) \chi^{\prime \prime}\left(\omega_{i j}\right)\right. \\
& \left.+\sum_{n}\left(M_{\mathrm{in}}^{2} \frac{\chi^{\prime \prime}\left(\omega_{\mathrm{in}}\right)}{e^{h \omega_{\mathrm{in}} / k_{\mathrm{B}} T}-1}+M_{n j}^{2} \frac{\chi^{\prime \prime}\left(\omega_{n j}\right)}{e^{h \omega_{n j} / k_{\mathrm{B}} T}-1}\right)\right]
\end{aligned}
$$

where $J_{\mathrm{ex}}$ is the exchange integral between the charge carriers and the felectrons of the $\mathrm{R}^{3+}$ ions and $M_{i j}$ the transition matrix element. $\chi^{\prime \prime}(\omega)$ is the same local susceptibility as used in nuclear relaxation. For a noninteracting Fermi liquid

$$
\chi^{\prime \prime}(\omega)=\pi[N(0)]^{2} \omega
$$

where $N(0)$ is the density of states at the Fermi energy. We immediately recognize that the linewidth of crystal-field excitations is zero in the superconducting state, since $N(0)$ vanishes due to the formation of Cooper pairs. This has been demonstrated in the first neutron spectroscopic study on a classical superconductor. The linewidth of the lowest-lying crystal-field transition observed for $\mathrm{La}_{1-x} \mathrm{~Tb}_{x} \mathrm{Al}_{2}$ [18] was indeed zero below $T_{c}$, raised exponentially around $T_{c}$, and followed the behavior described by Eq. (1) above $T_{c}$. Very recently a detailed study of the crystal-field linewidth was performed for the optimally doped high$T_{c}$ superconductor $\mathrm{Ho}_{0.1} \mathrm{Y}_{0.9} \mathrm{Ba}_{2} \mathrm{Cu}_{3} \mathrm{O}_{7-\delta}\left(T_{c}=92 \mathrm{~K}\right)$ [7]. The linewidth was observed to be zero only at the lowest temperatures, started to increase far below $T_{c}$, and converged into the Korringa behavior described by Eq. (1) above $T_{c}$. The unusual linewidth behavior below $T_{c}$. was ascribed to a high degree of gap anisotropy.

We have performed linewidth studies for the slightly underdoped high- $T_{6}$ superconductors $\mathrm{HoBa}_{2} \mathrm{Cu}_{4} \mathrm{O}_{8}\left(T_{c}=80 \mathrm{~K}\right)$ and $\mathrm{Er}_{2} \mathrm{Ba}_{4} \mathrm{Cu}_{7} \mathrm{O}_{15}\left(T_{c}=\right.$ $89 \mathrm{~K}$ ) by measuring crystal-field excitations at $h \omega \approx 1 \mathrm{meV}$ and $h \omega \approx 10 \mathrm{meV}$, respectively. The inelastic neutron scattering experiments were performed with use of the triple-axis spectrometer IN3 at the ILL. The temperature dependence of the observed linewidths is displayed in Fig. 3. Any deviation from the constant ratio $\Gamma_{\mathrm{obs}} / \Gamma_{i j}=1$ is a signature for the formation of Cooper pairs. The temperature dependence of the linewidths is highly unusual for both compounds. Firstly, the linewidths increase already far below $T_{c}$, consistent with a large gap anisotropy as observed for $\mathrm{H}_{0.1} \mathrm{Y}_{0.9} \mathrm{Ba}_{2} \mathrm{Cu}_{3} \mathrm{O}_{7-\delta}$ [7]. Secondly, the 


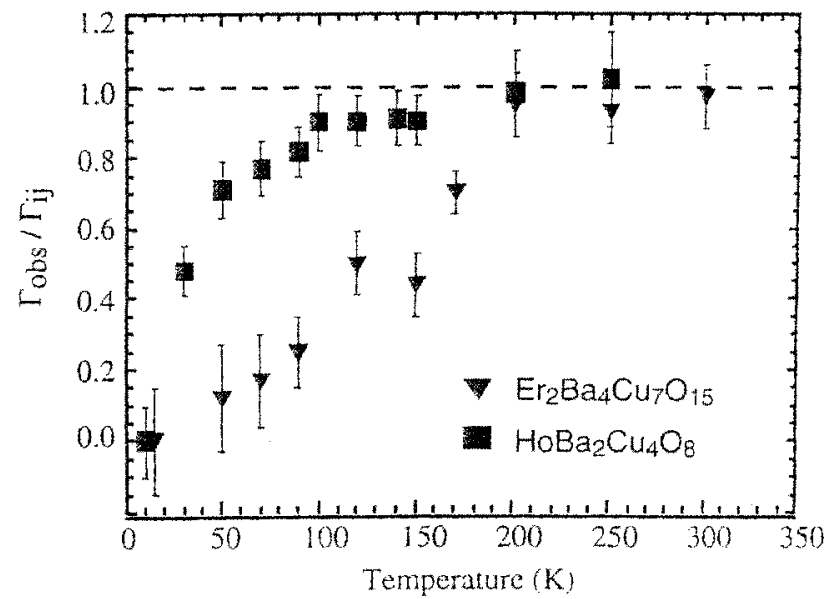

Fig. 3. Temperature variation of the reduced linewidths $\Gamma_{a b s} / \Gamma_{y}$ determined from crystal-field transitions observed for $\mathrm{HoBa}_{2} \mathrm{Cu}_{4} \mathrm{O}_{8}$ and $\mathrm{Er}_{2} \mathrm{Ba}_{4} \mathrm{Cu}_{7} \mathrm{O}_{15}$.

Korringa behavior described by Eq. (1) is only observed for temperatures $T>T^{*} \approx 200 \mathrm{~K}$, i.e., the systems have developed a pseudogap below which the density of states of the charge carriers is obviously suppressed. Thirdly, the relaxation behavior depends distinctly on the energy $h \omega$ at which we probe the local susceptibility $\chi^{\prime \prime}(\omega)$.

\section{CONCLUSIONS}

We have shown that neutron crystal-field spectroscopy is a valuable tool to investigate the inhomogeneous character of underdoped and overdoped high- $T_{c}$ superconductors. For underdoped systems, the origin of the inhomogeneities is believed to lie in the small hole density which gives rise to a strong enhancement of phase fluctuations [19]. A direct consequence of the phase fluctuations is the appearance of two crossovers (as observed by ARPES [8]. NMR [20], and neutron crystal-field [present work] experiments) which merge at the optimum doping level (see Fig. 4). At $T^{*}$ the systems develop a pseudogap below which the spectral weight of the charge carriers is suppressed, but only at $T_{c}<T^{*}$ is long-range phase coherence achieved, giving rise to bulk superconductivity. It has been suggested [20] that the pseudogap may be associated with the spin gap $E_{\mathrm{G}}$ observed in neutron investigations of the spin fluctuations $[21,22]$. However, the characteristic temperature where the spin gap opens is increasing with increasing doping, i.e., its temperature evolution is opposite to the behavior of the pseudogap temperature $T^{*}$, which means that the origin of the characteristic temperatures associated with the charge and spin excitations

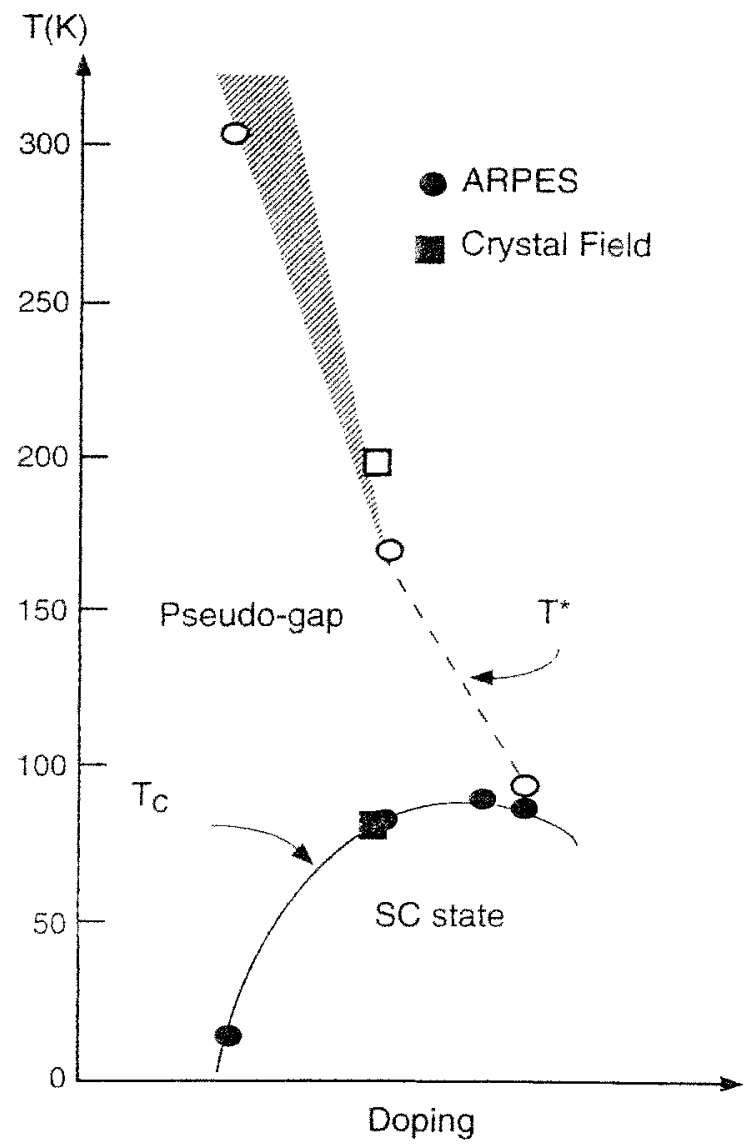

Fig. 4. Schematic phase diagram of high- $T_{c}$ superconductors as a function of doping. Full and empty symbols denote $T_{x}$ and $T^{*}$, respectively. Circles and squares stand for $\mathrm{Bi}_{2} \mathrm{Sr}_{2} \mathrm{CaCu}_{2} \mathrm{O}_{3+,}$ [8] and $\mathrm{HoBa}_{2} \mathrm{Cu}_{4} \mathrm{O}_{3}$ and $\mathrm{Er}_{2} \mathrm{Ba}_{4} \mathrm{Cu}_{7} \mathrm{O}_{15}$, respectively.

may be basically different. So far a detailed understanding of the connection between $T_{c}, T^{*}$, and $E_{\mathrm{G}}$ is missing.

\section{ACKNOWLEDGMENTS}

Financial support by the Swiss National Science Foundation is gratefully acknowledged.

\section{REFERENCES}

1. V. Hizhnyakov and E. Sigmund, Physica C 154, 655 (1988).

2. Phase Separation in Cuprate Superconductors, E. Sigmund and K. A. Müller, eds. (Springer, Berlin, 1994)

3. J. A. Hodges, P. Bonville, P. Imbert, and G. Jehanno, Physica C 184, 259 (1991).

4. Ch. Niedermayer, C. Bernhard, and J. I. Budnick, J. Magn Magn. Mater. 140-144, 1287 (1995)

5. M. A. Teplov, O. N. Bakharev, A. V. Dooglav, A. V. Egorov M. V. Eremin, M. S. Tagirov, A. G. Volodin, and R. Sh. Zhdanov, Physica C 185-189, 1107 (1991).

6. J. Röhler, P. Loeffen. S. Müllender, K. Conder, and E. Kaldis, this volume. 
7. A. T. Boothroyd, A. Mukherjee, and A. P. Murani, Phws. Rev. Lett. 77, $1600(1996)$

8. H. Ding. T. Yokoya. J. C. Campuzano, T. Takahashi, M. Randeria, M. R. Norman. T. Mochiku, K. Kadowaki, and J. Giapintzakis, Nature 382. 51 (1996).

9. P. Allenspach, A. Furrer, R. Osborn, and A. D. Taylor, Z. Pltys. B: Condens. Matter 85, 301 (1991)

10. W. Henggeler, J. Mesot, A. Furrer, G. Cuntze. M. Klauda, and G. Saemann-Ischenko, Europhys. Lett. 29, 233 (1995).

11. S. Kirkpatrick, Rev. Mod. Phys. 45, 574 (1973).

12. A. J. Sanjurjo, G. B. Martins, P. G. Pagliuso, E. Granado, I. Torriani, C. Rettori, S. Oseroff, and Z. Fisk, Phys. Rev. B 51 $1185(1995)$

13. J. Mesot, P. Allenspach, U. Staub, A. Furrer, H. Mutka. R. Osborn, and A. D. Taylor, Phys. Rev. B $47,6027(1993)$

14. J. Mesot, P. Allenspach, U. Staub, A. Furrer, and H. Mutka, Phys. Rev. Lett. 70, 865 (1993)
15. J.-Y. Genoud, T. Graf, A. Junod. D. Sanchez, G. Triscone, and J. Multer, Phusica C 177, 315 (199i).

16. G. Böttger, I. Mangelschots, E. Kaldis, P. Fischer, Ch. Krüger and F. Fauth, J. Phys.: Condens. Matter 8, 8889 (1996)

17. K. W. Becker, P. Fuide, and J. Keller, Z. Phys. B 28, 9 (1977).

18. R. Feile, M. Loewenhaupt, J. K. Kjems, and H. E. Hoenig, Phys, Ren, Lett. 47,610 (1981).

19. V. J. Emery and S. A. Kivelson. Nature 374, 434 (1995).

20. R. Stern, M. Mali, J. Roos, and D. Brinkmann, Phys Rev. $B$ 51. 15478 (1995).

21. J. Rossat-Mignod, L. P. Regnault, P. Bourges, P. Burlet. C. Vettier, and $\mathrm{Y}$. Henri, in Selected Topics in Superconductivity, L. C. Gupta and M. S. Multani, eds. (World Scientific, Singapore. 1993), pp. 265-347.

22. P. Dai, M. Yethiray, H. A. Mook, T. B. Lindemer, and F. Dogan. Phys. Rev. Lett. 77. 5425 (1996). 